\title{
Preliminary report on MFM measurements on magnetic nanofiber mats
}

\author{
Raphael Weiss, Andrea Ehrmann* \\ Bielefeld University of Applied Sciences, Faculty of Engineering and Mathematics, Bielefeld, Germany \\ ${ }^{*}$ Corresponding author E-mail address: andrea.ehrmann@fh-bielefeld.de
}

\section{INFO}

CDAPT, ISSN 2701-939X

Peer reviewed article

2021, Vol. 2, No. 1, pp. 1-7

DOI 10.25367/cdatp.2021.2.p1-7

Received: 13 December 2020

Accepted: 06 January 2021

Available online: 15 January 2021

\begin{abstract}
Nanofiber mats can be produced unambiguously by electrospinning. Besides pure polymers or polymer blends, such nanofibers can also contain metals, ceramics, etc., often introduced in the form of nanoparticles embedded in the spinning solution. Especially in case of magnetic nanoparticles, the physical properties of the whole nanofiber mats will strongly depend on the dispersion of the nanoparticles in the fibers - while small single nanoparticles may show superparamagnetic behavior, larger agglomerations will rather tend to showing ferromagnetic properties. Investigations of the magnetic properties of a sample with high spatial resolution are mostly performed by magnetic force microscopy (MFM). This technique, however, is usually applied on very flat surfaces of thin-film or nanostructured samples. Here, we report for the first time on MFM measurements on magnetic nanofiber mats, proving in principle that this technique can be used to investigate magnetic nanofiber mats, while the highly uneven nanofiber structure still causes large problems which have to be solved in the future.
\end{abstract}

\section{Keywords}

atomic force microscopy (AFM), magnetic force microscopy (MFM), electrospinning, magnetic nanofiber mats, magnetite
(C) 2021 The authors. Published by CDAPT.

This is an open access article under the CC BY-NC-ND license https://creativecommons.org/licenses/ peer-review under responsibility of the scientific committee of the CDAPT.

\section{Introduction}

Nanofiber mats are typically produced by electrospinning, i.e. by creating fibers with diameters of some ten to some hundred nanometers in a strong electric field from a polymer solution or melt [1-3]. Such nanofiber mats can be used for diverse applications in which their large surface-to-volume ratio is important, such as wound dressings [4], tissue engineering [5,6], catalyzers [7], or filters [8-10]. Besides man-made polymers [11,12] and biopolymers [13,14], polymers blended with non-solvable materials can also be used for electrospinning [15,16]. 
In this way, it is also possible to prepare magnetic nanofiber mats for diverse applications [17-19]. Such magnetic nanofibers are also highly interesting for basic research and future applications in spintronics or neuromorphic computing [20-24].

For both basic and applied research, however, it is necessary to characterize the distribution of magnetic nanoparticles inside the fibers. It was shown experimentally and in simulations that modifications of average distances between the nanoparticles, either due to fiber shrinkage during carbonization [25] or due to agglomerations [26], significantly modify the magnetic properties of such nanofibers.

While transmission electron microscopy (TEM) offers the possibility to "look inside" nanofibers and to investigate the nanoparticle distribution in nanofiber mats [26], this method is highly time-consuming, complicated and necessitates expensive equipment which is not available in each university of research institute. It would be ideal to directly map the magnetic properties of such nanofiber mats with sufficient spatial resolution.

A technique which can indeed be used for this purpose is magnetic force microscopy (MFM). Opposite to atomic force microscopy (AFM) which uses a cantilever to detect a sample's topography and possibly some additional mechanical properties of the surface, the MFM uses a magnetized cantilever tip and can thus be applied in a special software mode, allowing for measuring magnetic properties in addition to the sample's topography [27]. There are even a few reports in the literature showing MFM measurements on single magnetic nanofibers or nanowires attached to flat substrates [28-32]. To the best of our knowledge, however, no report was published before on MFM measurements on completely uneven, irregular magnetic nanofiber mats.

Here we report on first results of such MFM measurements on different electrospun magnetic nanofiber mats, prepared with magnetite or nickel-ferrite. We show that MFM can in principle be used to investigate magnetic properties of magnetic nanofiber mats, but further research is necessary to gain clearer images of the spatially resolved magnetic properties.

\section{Experimental}

Nanofiber mats were electrospun using the wire-based system "Nanospider Lab" (Elmarco, Liberec, Czech Republic) with the following spinning parameters: voltage $80 \mathrm{kV}$, nozzle diameter $0.9 \mathrm{~mm}$, carriage speed $150 \mathrm{~mm} / \mathrm{s}$, distance between lower electrode and substrate $240 \mathrm{~mm}$, distance between substrate and upper electrode $50 \mathrm{~mm}$, relative humidity in the chamber $32 \%$, temperature $22{ }^{\circ} \mathrm{C}$.

Spinning solutions were prepared from $14 \%$ polyacrylonitrile (PAN) (X-PAN, Dralon, Dormagen, Germany) dissolved in dimethyl sulfoxide (DMSO) ( $\min 99.9 \%$, purchased from S3 chemicals, Bad Oeynhausen, Germany). $\mathrm{Fe}_{3} \mathrm{O}_{4}$ (magnetite) nanoparticles (50-10 nm diameter, Merck KGaA, Darmstadt, Germany) were either applied by dip-coating from an aqueous solution after electrospinning, or added to the spinning solution in a weight ratio polymer:nanoparticles of 1:1.8, which was the largest nanoparticle concentration found spinnable in an earlier investigation [24,25]. Directly before electrospinning, the dispersion was ultrasonically stirred for $40 \mathrm{~min}$ at $35{ }^{\circ} \mathrm{C}$ with a frequency of $37 \mathrm{kHz}$ to avoid agglomerations mostly. It should be mentioned that the distribution of the nanoparticles in the fibers is not homogeneous [26], thus no perfect magnetic signals along the whole nanofiber mat can be expected, but parts with strong signals due to agglomerations and completely nonmagnetic parts.

As a reference of a perfectly flat magnetic sample, a cobalt (Co) layer (25 $\mathrm{nm}$ thickness) on a silicon (Si) wafer was used. In Co thin films, former investigations found circular and elliptical magnetic bubbles at the remanent state (i.e. without external magnetic field) after saturation [33], so that some magnetic pattern can be expected to be visible here.

AFM images were taken by a FlexAFM Axiom (Nanosurf, Liestal, Switzerland), equipped with a cantilever MagneticMulti75-G (NanoAndMore $\mathrm{GmbH}$, Wetzlar, Germany) and post-processed with Gwyddion 2.51. 


\section{Results and discussion}

Firstly, Fig. 1 shows an AFM topography image and the correlated magnetic MFM image of the flat reference sample. While the topography (Fig. 1a) is nearly flat, besides some fine grains of dust, a clear structure is visible in the MFM images (Fig. 1b) which can be attributed to the magnetic properties of the thin-film sample. Such MFM images are often scaled in black-and-white to enhance the contrast; however, here we decided to use a similar contrast as typical for the topography to allow for seeing all features available in the sample on these relatively large scales.
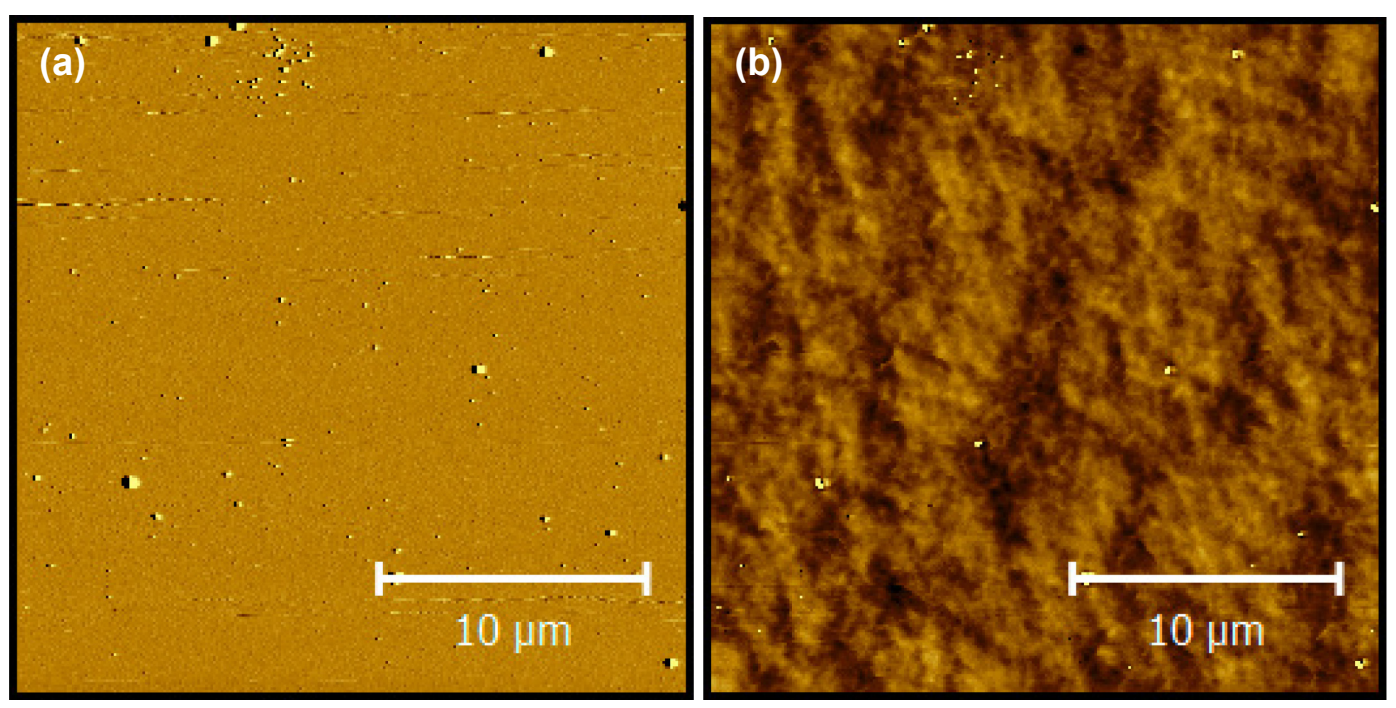

Fig. 1 (a) AFM topography image of a magnetic thin-film sample; (b) MFM phase showing the magnetic properties of the sample

Investigating nanofiber mats with MFM, however, is significantly more complicated since now topography and magnetic properties mix and have to be separated carefully. Fig. 2a shows the topography of a carbonized magnetic nanofiber mat, with some typical errors occurring when the tip touches the fibers, as it happens often when measuring such non-continuous surfaces.
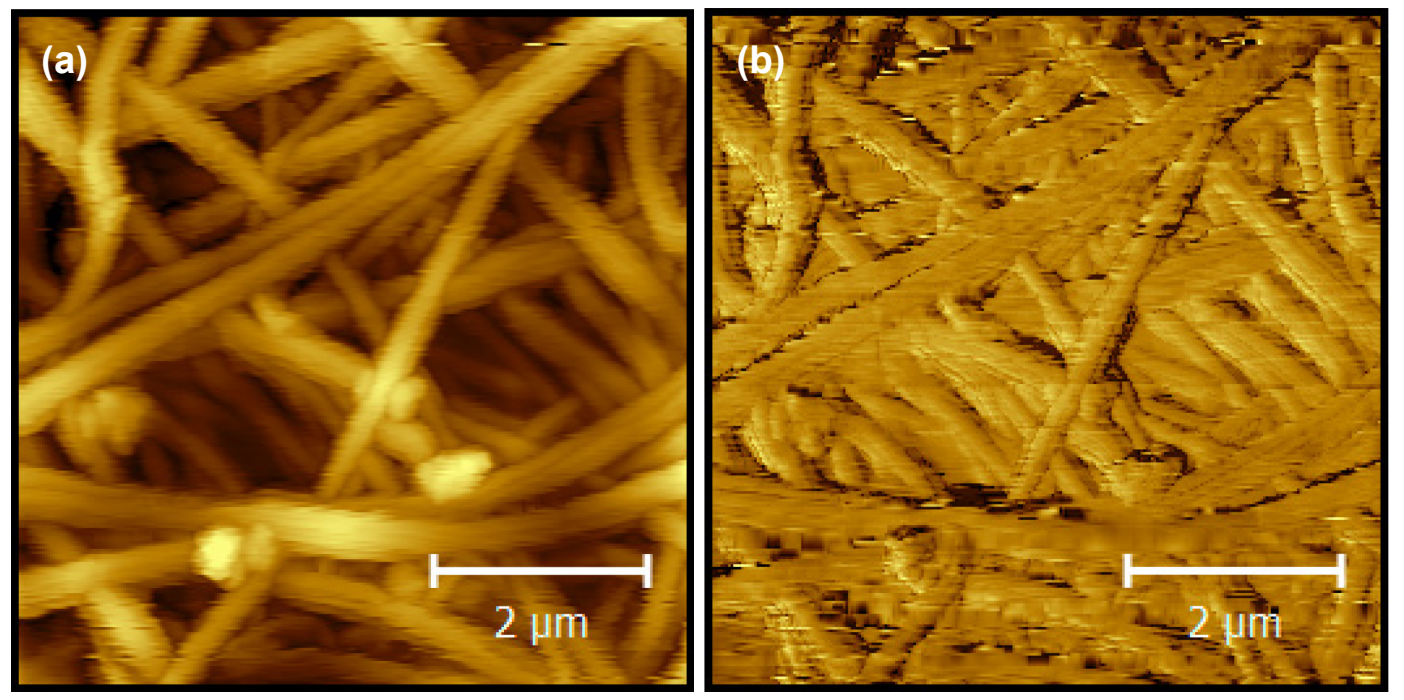

Fig. 2 (a) AFM topography image of a carbonized magnetic nanofiber mat; (b) MFM phase showing the magnetic properties of the sample (tip lift height $30 \mathrm{~nm}$ )

The MFM phase depicted in Fig. 2b, however, mostly reflects this topography, although the "contour following" mode should mostly suppress this problem. 
MFM measurements are performed in a double-scan process, e.g. by measuring the lowest line in the image from left to right to detect the topography, and then scanning the same path back with the cantilever lifted by a certain distance (here $30 \mathrm{~nm}$ ) to detect mostly the magnetic force. Alternatively, the so-called "dual pass mode" can be used, meaning that two firstly the topography is scanned from left to right and back, and afterwards the same line is scanned from left to right and back in MFM mode, i.e. with lifted tip, making the image more accurate, but doubling the time for measuring. Generally, in MFM it is necessary to find a compromise between smaller heights during the MFM scanning time, increasing the influence of the topography and making the signals harder to interpret, and larger heights, decreasing the resolution. This is especially important in case of strongly structured surfaces, such as nanofiber mats. Here, apparently, the chosen height was not large enough so that no clear magnetic signal can be observed.

Next, samples were investigated with larger distances. Fig. 3 shows an example of a measurement with a tip lifting height of $170 \mathrm{~nm}$ during the MFM scanning time. Indeed, the topography becomes nearly invisible here, now allowing for evaluating the magnetic information. While this would be acceptable for large magnetic structures, as visible in Fig. $1 \mathrm{~b}$, it is not sufficient for the investigation of magnetic nanoparticles embedded in (or coated on) nanofibers. Thus, the next step is an optimization of the tip lifting height to enable sufficiently resolved magnetic scans of these challenging samples.
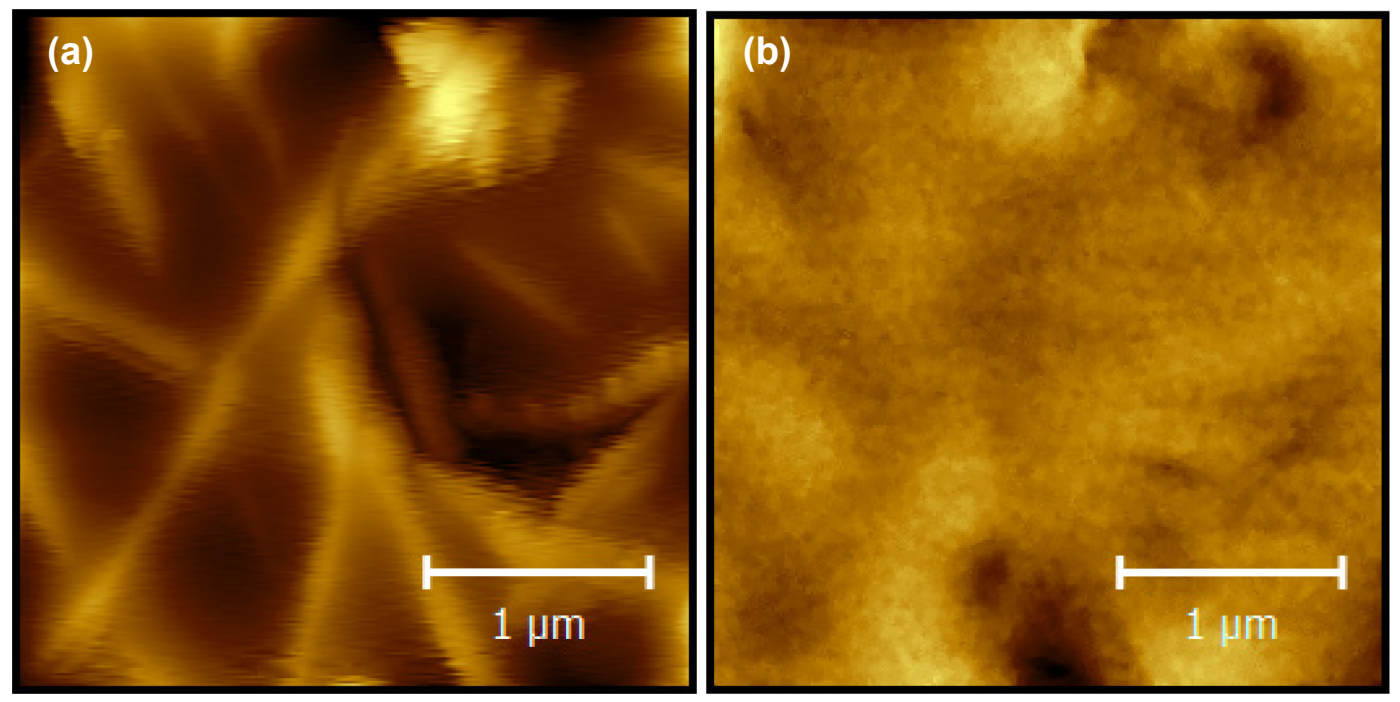

Fig. 3 (a) AFM topography image of a magnetically coated nanofiber mat; (b) MFM phase showing the magnetic properties of the sample (tip lift height $170 \mathrm{~nm}$ )

As an example for the influence of the tip lifting height, Fig. 4 shows measurements on the coated nanofiber mat, now again with a slightly reduced magnification, for lift heights of $300 \mathrm{~nm}$ (Fig. 4a) and $240 \mathrm{~nm}$ (Fig. 4b), respectively.

It is clearly visible that the magnetic structure becomes clearer for the lower tip lift height; but even for the relatively large lift heights chosen here, the nanofibers start becoming visible again. This example shows that while the magnetic features, here visible as large dark and bright areas, can indeed be visualized by MFM, but underline also that in spite of using the "contour follow" mode which should significantly reduce the influence of the topography, the latter cannot be ignored in magnetic investigations of electrospun nanofiber mats.

It must also be mentioned that optimizing the tip lift height is only possible for a specific situation. Fig. 5 shows the same nanofiber mat as depicted in Fig. 4 after slightly shifting the measurement position, measured with identical magnification and measurement parameters as well as with an intermediate tip lift height of $280 \mathrm{~nm}$. Here, however, the topography is much stronger visible than in Fig. 4. This shows again that there is still a long way to go from this first proof-of-principle to a reliable technique which can be used to detect magnetic nanoparticles inside magnetic nanofiber mats. 


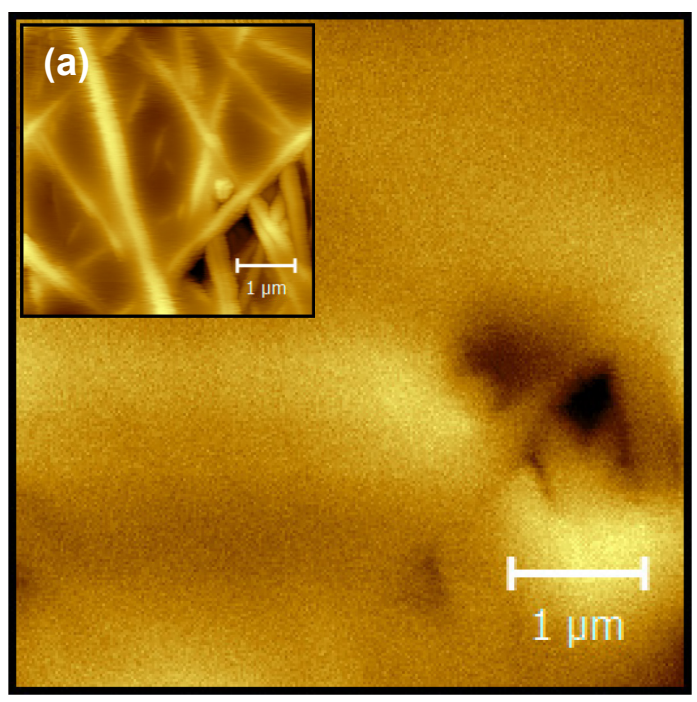

(b)

Fig. 4 MFM phases of a magnetically coated nanofiber mat with different tip lift heights: (a) $300 \mathrm{~nm}$; (b) $240 \mathrm{~nm}$. The inset shows the AFM topography.
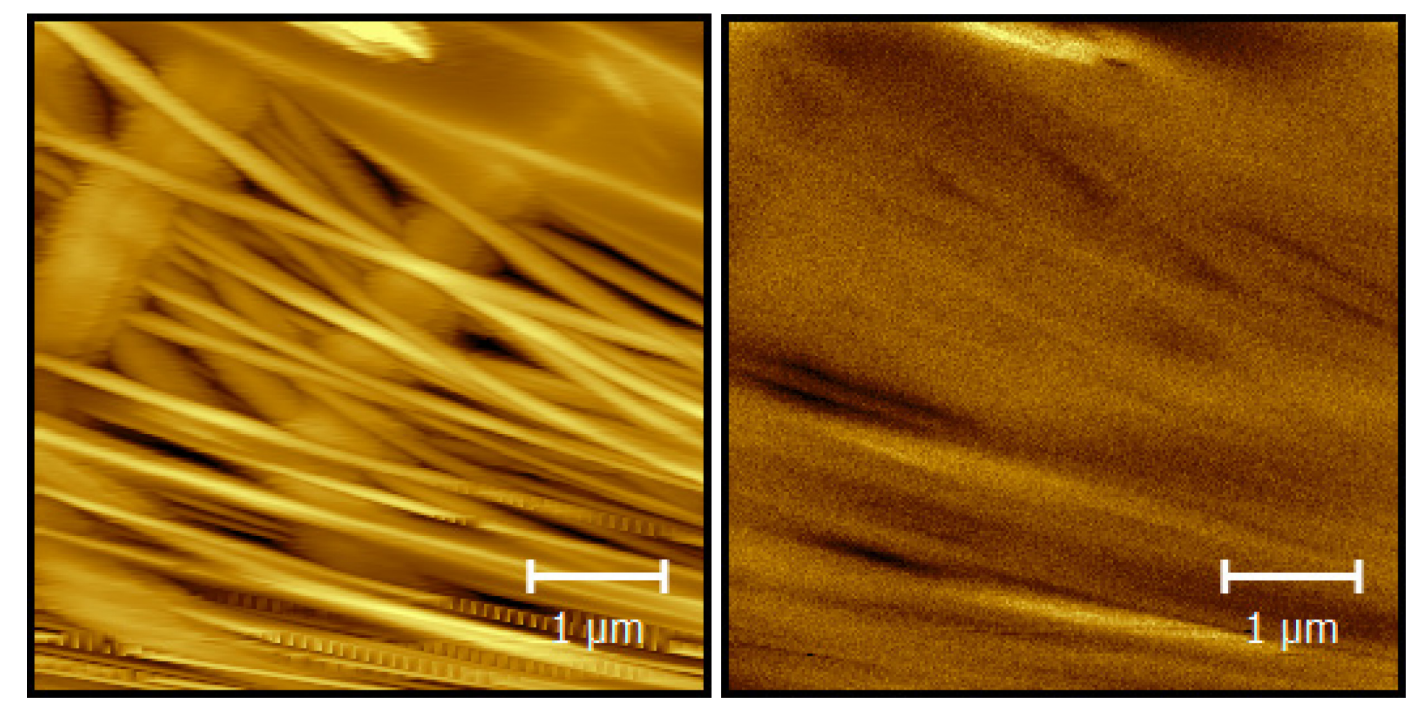

Fig. 5 (a) AFM topography image of a magnetically coated nanofiber mat; (b) MFM phase showing the magnetic properties of the sample (tip lift height $280 \mathrm{~nm}$ )

Besides, several further questions, especially related to the possible dimensions of nanoparticle agglomerations which are directly connected with their magnetic properties [26], must be investigated to develop this technique further and to enable a reliable interpretation of MFM images of magnetic nanofiber mats.

\section{Conclusions}

While magnetic force microscopy (MFM) is a typical tool for the evaluation of magnetic thin-film samples or nanostructures on flat surfaces, no previous attempts were found in the literature to use MFM for the investigation of magnetic nanofiber mats. Here we present first MFM measurements on such magnetic nanofiber mats, showing the general suitability of this method to investigate magnetic nanofiber mats, but also the challenges which have to be taken into account in these measurements.

Since no literature exists yet on measuring MFM on magnetic nanofiber mats, it is necessary to optimize the measurements without drawing on previous experience of other groups. Thus, as the next step it is planned to electrospin aligned magnetic nanofibers [34] to reduce the roughness und to optimize MFM 
process parameters for this situation, before transferring this optimization back to isotropic (not oriented) magnetic nanofiber mats.

\section{References}

[1] T. Subbiah, G. S. Bhat, R. W. Tock, S. Parameswaran, S. S. Ramkumar. 2005. Electrospinning of nanofibers. J. Appl. Polymer Sci. 96, 2, 557-569. DOI: https://doi.org/10.1002/app.21481

[2] D. Li, Y. Xia. 2004. Electrospinning of nanofibers: reinventing the wheel? Adv. Mater. 16, 14, 1151-1170. DOI: https://doi.org/10.1002/adma.200400719

[3] A. Greiner, J. H. Wendorff. 2007. Electrospinning: a fascinating method for the preparation of ultrathin fibers. Angew. Chem. Int. Ed. 46, 30, 5670-5703. DOI: https://doi.org/10.1002/anie.200604646

[4] A. Mamun. 2019. Review of possible applications of nanofibrous mats for wound dressings. Tekstilec 62, 2, 89-100. DOI: https://doi.org/10.14502/Tekstilec2019.62.89-100

[5] N. Ashammakhi, A. Ndreu, Y. Yang, H. Ylikauppila, L. Nikkola. 2012. Nanofiber-based scaffolds for tissue engineering. Eur. J. Plast. Surg. 35, 2, 135-149. DOI: https://doi.org/10.1007/s00238-008-0217-3

[6] J. Bockelmann, K. Klinkhammer, A. von Holst, N. Seiler, A. Faissner, G. A. Brook, D. Klee, J. Mey. 2011. Functionalization of electrospun poly( $\varepsilon$-caprolactone) fibers with the extracellular matrix-derived peptide GRGDS improves guidance of Schwann cell migration and axonal growth. Tissue Eng. A 17, 3-4, 475-486. DOI: https://doi.org/10.1089/ten.tea.2010.0369

[7] X. Wang, Y. G. Kim, C. Drew, B. C. Ku, J. Kumar, L. A. Samuelson. 2004. Electrostatic assembly of conjugated polymer thin layers on electrospun nanofibrous membranes for biosensors. Nano Lett. 4, 2, 331334. DOI: https://doi.org/10.1021/nl034885z

[8] B. Yalcinkaya, F. Yalcinkaya, J. Chaloupek. 2016. Thin film nanofibrous composite membrane for dead-end seawater desalination. J. Nanomater. 2016, 2694373. DOI: https://doi.org/10.1155/2016/2694373

[9] R. Roche, F. Yalcinkaya. 2019. Electrospun polyacrylonitrile nanofibrous membranes for point-of-use water and air cleaning. ChemistryOpen 8, 97-103. DOI: https://doi.org/10.1002/open.201800267

[10] R. Torres-Mendieta, F. Yalcinkaya, E. Boyraz, O. Havelka, W. Waclawek, J. Maryska, M. Cerník, M. Bryjak. 2020. PVDF nanofibrous membranes modified via laser-synthesized Ag nanoparticles for a cleaner oily water separation. Appl. Surf. Sci. 526, 146575. DOI: https://doi.org/10.1016/j.apsusc.2020.146575

[11] J. F. Pan, N. H. Liu, H. Sun, F. Xu. 2014. Preparation and characterization of electrospun PLCL/poloxamer nanofibers and dextran/gelatin hydrogels for skin tissue engineering. PLOS ONE 9, e112885. DOI: https://doi.org/10.1371/journal.pone.0112885

[12] T. Grothe, D. Wehlage, T. Böhm, A. Remche, A. Ehrmann. 2017. Needleless electrospinning of PAN nanofiber mats. Tekstilec 60, 290-295. DOI: https://doi.org/10.14502/Tekstilec2017.60.290-2

[13] T. Maver, M. Kurecic, D. M. Smrke, K. S. Kleinschek, U. Maver. 2016. Electrospun nanofibrous CMC/PEO as a part of an effective pain-relieving wound dressing. J. Sol-Gel Sci. Technol. 79, 475-486. DOI: https://doi.org/10.1007/s10971-015-3888-9

[14] B. Ebrahimi-Hosseinzadeh, M. Pedram, A. Hatamian-Zarmi, S. Salahshour-Kordestani, M. Rasti, Z. B. Mokhtari-Hosseini, M. Mir-Derikvand. 2016. In vivo evaluation of gelatin/hyaluronic acid nanofiber as burnwound healing and its comparison with ChitoHeal gel. Fibers Polym. 17, 820-826. DOI: https://doi.org/10.1007/s12221-016-6259-4

[15] K.-H. Na, W.-T. Kim, D.-C. Park, H. G. Shin, S. H. Lee, J. S. Park, T. H. Song, W. Y. Choi. 2018. Fabrication and characterization of the magnetic ferrite nanofibers by electrospinning process. Thin Sol. Films 660, 358364. DOI: https://doi.org/10.1016/j.tsf.2018.06.018

[16] R. J. R. Matos, C. I. P. Chaparro, J. C. Silva, M. A. Valente, J. P. Borges, P. I. P. Soares. 2018. Electrospun composite cellulose acetate/iron oxide nanoparticles non-woven membranes for magnetic hyperthermia applications. Carbohydr. Polym. 198, 9-16. DOI: https://doi.org/10.1016/j.carbpol.2018.06.048

[17] H. H. Liu, Y. J. Li, M. W. Yuan, G. B. Sun, Q. L. Liao, Y. Zhang. 2018. Solid and macroporous $\mathrm{Fe}_{3} \mathrm{C} / \mathrm{N}-\mathrm{C}$ nanofibers with enhanced electromagnetic wave absorbability. Sci. Rep. 8, 16832. DOI: https://doi.org/10.1038/s41598-018-35078-z

[18] K.-Y. A. Lin, M.-T. Yang, J.-T. Lin, Y. C. Du. 2018. Cobalt ferrite nanoparticles supported on electrospun carbon fiber as a magnetic heterogeneous catalyst for activating peroxymonosulfate. Chemosphere 208, 502511. DOI: https://doi.org/10.1016/j.chemosphere.2018.05.127

[19] Y. Q. Zhan, Z. H. Long, X. Y. Wan, J. M. Zhang, S. J. He, Y. He. 2018. 3D carbon fiber mats/nano- $\mathrm{Fe}_{3} \mathrm{O}_{4}$ hybrid material with high electromagnetic shielding performance. Appl. Surf. Sci. 444, 710-720. DOI: https://doi.org/10.1016/j.apsusc.2018.03.006

[20] K.-S. Ryu, L. Thomas, S.-H. Yang, S. S. P. Parkin. 2012. Appl. Current induced tilting of domain walls in high velocity motion along perpendicularly magnetized micron-sized Co/Ni/Co racetracks. Phys. Expr. 5, 093006. DOI: https://doi.org/10.1143/APEX.5.093006

[21] O. Alejos, V. Raposo, L. S. Tejerina, E. Martinez. 2017. Efficient and controlled domain wall nucleation for magnetic shift registers. Sci. Rep. 7, 11909. DOI: https://doi.org/10.1038/s41598-017-12230-9 
[22] C. Garg, S.-H. Yang, T. Phung, A. Pushp, S. S. P. Parkin. Dramatic influence of curvature of nanowire on chiral domain wall velocity. Sci. Adv. 3, e1602804. DOI: https://doi.org/10.1126/sciadv.1602804

[23] T. Blachowicz, A. Ehrmann. 2018. Magnetization reversal in bent nanofibers of different cross-sections. J. Appl. Phys. 124, 152112. DOI: https://doi.org/10.1063/1.5022065

[24] C. Döpke, T. Grothe, P. Steblinski, M. Klöcker, L. Sabantina, D. Kosmalska, T. Blachowicz, A. Ehrmann. 2019. Magnetic nanofiber mats for data storage and transfer. Nanomater, 9, 92. DOI: https://doi.org/10.3390/nano9010092

[25] N. Fokin, T. Grothe, A. Mamun, M. Trabelsi, M. Klöcker, L. Sabantina, C. Döpke, T. Blachowicz, A. Hütten, A. Ehrmann. 2020. Magnetic properties of electrospun magnetic nanofiber mats after stabilization and carbonization. Materials 13, 7, 1552. DOI: https://doi.org/10.3390/ma13071552

[26] M. Wortmann, A. S. Layland, N. Frese, U. Kahmann, T. Grothe, J. L. Storck, T. Blachowicz, J. Grzybowski, B. Hüsgen, A. Ehrmann. 2020. On the reliability of highly magnified micrographs for structural analysis in materials science. Sci. Rep. 10, 14708. DOI: https://doi.org/10.1038/s41598-020-71682-8.

[27] J. I. Martín, J. Nogués, K. Liu, J. L. Vicent, Ivan K. Schuller. 2003. Ordered magnetic nanostructures: fabrication and properties. J. Magn. Magn. Mater. 256, 1-3, 449-501. DOI: https://doi.org/10.1016/S03048853(02)00898-3

[28] M. Donolato, C. Tollan, J. M. Porro, A. Berger, P. Vavassori. 2013. Flexible and stretchable polymers with embedded magnetic nanostructures. Adv. Mater. 25, 4, 623-629. DOI: https://doi.org/10.1002/adma.201203072

[29] K. Prashanthi, P. M. Shaibani, A. Sohrabi, T. S. Natarajan, T. Thundat, T. Nanoscale magnetoelectric coupling in multiferroic $\mathrm{BiFeO}_{3}$ nanowires. Phys. Stat. Sol. RRL 6, 6, 244-246. DOI: https://doi.org/10.1002/pssr.201206135

[30] K. Prashanthi, T. Thundat. 2014. In situ study of electric field-induced magnetization in multiferroic $\mathrm{BiFeO}_{3}$ nanowires. Scanning 36, 224-230. DOI: https://doi.org/10.1002/sca.21092

[31] S. Choopani, F. Samavat, E. N. Kolobova, A. M. Grishin. 2020. Ferromagnetic resonance and magnetic anisotropy in biocompatible $\mathrm{Y}_{3} \mathrm{Fe}_{5} \mathrm{O}_{12} @ \mathrm{Na}_{0.5} \mathrm{~K}_{0.5} \mathrm{NbO}_{3}$ core-shell nanofibers. Ceramics International 46, 20722078. DOI: https://doi.org/10.1016/j.ceramint.2019.09.187

[32] N. Liu, P. C. Du, P. Zhou, R. G. Tanguturi, Y. J. Qi, T. J. Zhang. Magnetoelectric coupling in $\mathrm{CoFe}_{2} \mathrm{O}_{4-}$ $\mathrm{Pb}\left(\mathrm{Zr}_{0.2} \mathrm{Ti}_{0.8}\right) \mathrm{O}_{3}$ coaxial nanofibers. J. Am. Ceram. Soc. 00, 1-7. DOI: https://doi.org/10.1111/jace.17494

[33] A. Lisfi, J. C. Lodder. 2001. Magnetic domains in Co thin films obliquely sputtered on a polymer substrate. Phys. Rev. B 63, 174441. DOI: https://doi.org/10.1103/PhysRevB.63.174441

[34] J. L. Storck, T. Grothe, A. Mamun, L. Sabantina, M. Klöcker, T. Blachowicz, A. Ehrmann. 2020. Orientation of electrospun magnetic nanofibers near conductive areas. Materials 13, 47. DOI: https://doi.org/10.3390/ma13010047 